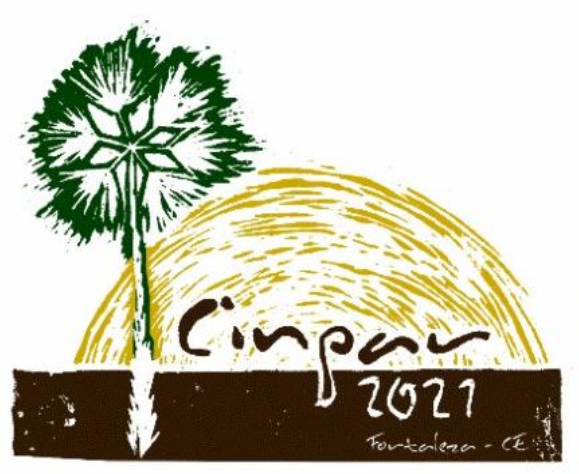

XVII Congresso Internacional sobre Patologia e

Reabilitação das Construções

XVII Congreso Internacional sobre Patología y Rehabilitación de las Construcciones

XVII International Conference on Pathology and Constructions Rehabilitation

FORTALEZA (Brasil), 3 a 5 de junho de 2021

https://doi.org/10.4322/CINPAR.2021.097

\title{
Vigas de Gran Altura de Hormigón Reforzado con Fibras. Evaluación de la Resistencia al Corte
}

\author{
Viviana C. ROUGIER ${ }^{1,2}$, Miqueas C. DENARDI ${ }^{1,2}$, Darío O. VERCESI ${ }^{2}$ \\ ${ }^{1}$ Universidad Tecnológica Nacional, Facultad Regional Concepción del Uruguay, Argentina, rougierv@frcu.utn.edu.ar, \\ denardim@frcu.utn.edu.ar \\ 2 Universidad Tecnológica Nacional, Facultad Regional Concordia, Argentina, dariovercesi@gmail.com
}

\begin{abstract}
Concrete is very strong in compression, but it has a very low tensile strength. To improve its tensile strength, reinforcing steel is often used in the concrete. However, the reinforcement of the cementitious matrix with discrete fibers has gained increasing recognition. The addition of fibers randomly distributed as reinforcement of cement-based matrices can produce a material with improved tensile strength and deformational characteristics. Different types of fibers can be employed to reinforce concrete. Nevertheless, the use of steel fibers is particularly attractive in concrete members with high reinforcement congestion, like deep beams, when conventional stirrups can be eliminated or reduced. So, the effects of steel fibers on the shear strength of reinforced concrete deep beams were evaluated by different ways: experimental, theoretical, and numerical. A total of six beams were subjected to a concentrated load P at their center and two steel fiber volume fractions were used. Two specimens were elaborated with plain concrete and longitudinal steel reinforcement. Web reinforcement was used in one of those beams and the other was made without stirrups. The others four specimens were built with steel fibers reinforced concrete (SFRC), longitudinal steel reinforcement and without stirrups. The test results indicated that the fibers influenced the shear strength of reinforced concrete deep beams. Shear strength increased with increasing fiber volume fraction, but steel fibers could not totally replace the conventional steel stirrups. Comparisons between experimental shear strength values and predictions, using empirical models developed by different authors, showed satisfactory results. In addition, the comparison between numerical and experimental values indicated that finite element analysis (FEA) was a reliable tool to simulate nonlinear behavior of SFRC deep beams.
\end{abstract}

Keywords: SFRC, deep beams, shear strength, FE analysis.

\section{Introducción}

El hormigón es un material muy versátil que posee buena resistencia a la compresión, pero su resistencia a tracción es baja. El uso de fibras dispersas en la matriz de hormigón es una opción que permite incrementar dicha resistencia. De este modo y teniendo en cuenta sus componentes, se puede definir el hormigón reforzado con fibras (HRF) como "un hormigón hecho principalmente de cemento portland, agregados y refuerzo de fibras discretas", ACI 544.1R (1996-2002). Entre las fibras que se pueden usar se encuentran las de acero, vidrio, polímeros orgánicos y en menor medida, las fibras vegetales, ACI 544.1R (1996-2002), siendo las primeras las que más se utilizan.

La incorporación de fibras de acero mejora la ductilidad, la resistencia a flexión, la capacidad para soportar cargas en estado fisurado, la tenacidad y capacidad para mantener la integridad luego de alcanzada la carga de rotura. La mejora en dichas propiedades depende de la cantidad, tipo, tamaño, resistencia y configuración de la fibra y están ligadas al proceso mediante el cual la carga es transmitida de la matriz a las 
fibras y el efecto de costura de fisuras, Ruano (2013). Sin embargo, se debe decir que las fibras de acero mejoran las propiedades mecánicas del hormigón no como reemplazo de la armadura de barras sino como complemento de éstas. Las barras de acero no pueden impedir a formación de micro fisuras, pero las fibras dispersas pueden prevenir la propagación o su crecimiento en ancho controlando el proceso de fisuración, Shah y Ribakov (2011). Por ello, el uso de dichas fibras, como complemento o reemplazo parcial de la armadura convencional de estribos, resulta de gran interés particularmente en el caso de vigas de gran altura, donde suelen existir grandes congestionamientos de armaduras. Algunas investigaciones han demostrado que el uso de hormigón reforzado con fibras de acero mejora significativamente el comportamiento de vigas de gran altura, proporcionando un incremento en la capacidad de carga y la ductilidad y un mejor control de la fisuración, aún para bajos contenidos de fibras: Beshara et al. (2015); Naik y Kute (2017); Pabale y Mehetre (2015); Moradi y Reza Esfahani (2017).

Las vigas de gran altura (VGA) son estructuras en forma de placas solicitadas en su plano y sustentadas como vigas. Se utilizan en numerosas estructuras tales como fundaciones, estructuras offshore, tanques, etc. También se suelen usar como elementos de transición entre columnas de niveles superiores a plantas inferiores de edificios elevados, cuando por razones arquitectónicas o funcionales son necesarias luces mayores entre columnas, Orler y Donini (2011). Debido a la pequeña relación entre la luz de corte y la altura útil $(\mathrm{a} / \mathrm{d})$, las VGA desarrollan un mecanismo de transferencia de carga diferente al de las vigas esbeltas. En las VGA, una gran proporción de la carga que soportan es transmitida directamente a los apoyos. Por ello las tensiones de corte son significativamente mayores, y en general controlan la resistencia de estos elementos. En la zona entre los apoyos y el punto de aplicación de la carga puede ocurrir la falla del hormigón por compresión, Ahmad et al. (2011).

Entre los procedimientos para el diseño de VGA se pueden mencionar el método de elementos finitos, la teoría de chapas utilizando la función de Airy o bien el método puntal- tensor adoptado por el Reglamento Argentino de Estructuras de Hormigón y diferentes códigos a nivel mundial. El "método puntal-tensor" (MPT), permite representar con un aceptable grado de aproximación, el comportamiento de aquellas zonas particulares de las estructuras en donde no es válida la hipótesis de Bernoulli, y, por lo tanto, no se cumple con la distribución lineal de las deformaciones en la sección o zona de estudio, Orler y Donini (2008).

EI MPT está basado en el teorema de límite inferior del cálculo plástico según el cual, si para una determinada carga se halla una distribución de tensiones de tal forma que en ningún punto se supere la tensión de plastificación de los materiales, se satisfaga el equilibrio estático y las condiciones de contorno, la estructura resistirá dicha carga sin llegar a la rotura. De este modo, la capacidad resistente obtenida con el método puntal tensor siempre es menor que la real, es decir la carga hallada es menor que la de colapso de la estructura, Adrija et al. (2017). Por ello algunos investigadores, en búsqueda de resultados más cercanos a la carga última han realizado modificaciones al MPT, entre ellos, Zhang y Tan (2007); Arabzadeh et al. (2009); Chetchotisak et al. (2014); Adrija et al. (2017); Chen et al. (2018).

Por otro lado, la aparición de los métodos computacionales, entre ellos el método de elementos finitos (MEF), y de software específicos de diseño, ha facilitado el cálculo, haciendo posible la determinación de los esfuerzos a los que se encuentra sometida una VGA; así como también la distribución de tensiones y deformaciones en dicho elemento.

En este trabajo se presentan los resultados de una campaña experimental tendiente a evaluar la efectividad del uso de HRFA en VGA en reemplazo de la armadura convencional de estribos, en términos de resistencia a corte, ductilidad y modos de falla. Se analizan dos fracciones de volúmenes de fibra, y la existencia o no de estribos horizontales y verticales. Todos los especímenes se ensayan a flexión en tres puntos. Se obtienen cargas máximas, y curvas carga-desplazamiento y se comparan los resultados experimentales con los valores de cargas últimas obtenidos de modelos teóricos. Además, lleva a cabo un análisis numérico usando un modelo no lineal de elementos finitos implementado en el software ABAQUS ${ }^{R}$. Los resultados se comparan con los datos experimentales demostrando que el análisis por elementos finitos es una herramienta efectiva y confiable para simular el comportamiento no lineal de VGA de HRFA. 


\section{Programa experimental}

\subsection{Generalidades}

El programa experimental se llevó a cabo mediante ensayos a flexión de VGA, cargadas con una fuerza vertical centrada y diseñadas siguiendo los lineamientos del CIRSOC 201-05, Apéndice A, CIRSOC 201 (2005). Se elaboraron seis vigas y dos tipos de hormigón, hormigón simple y HRFA. Dos vigas se fabricaron con hormigón simple y armadura de flexión, sin armadura de corte en un caso, y con armadura mínima de corte, en otro. Los restantes especímenes se elaboraron con HRFA, según dos fracciones de volumen de fibras, $0.31 \%\left(25 \mathrm{~kg} / \mathrm{m}^{3}\right)$ y $0.64 \%\left(50 \mathrm{~kg} / \mathrm{m}^{3}\right)$, y $\sin$ armadura de corte. En las Figuras 1 a y b, respectivamente, se pueden ver los detalles constructivos de un espécimen con armadura de corte y de otro con armadura de flexión solamente. Se adoptó un recubrimiento de $20 \mathrm{~mm}$, valor mínimo requerido para este tipo de estructuras por el CIRSOC 201-05 (2005). En la Tabla 1 se presenta la descripción de los especímenes ensayados en cuanto a denominación, dimensiones, cuantías de armaduras longitudinal $(\rho)$ y de estribos horizontales y verticales $\left(\rho_{h}\right.$ y $\left.\rho_{v}\right)$ y fracción de volumen de fibras $\left(V_{f}\right)$.

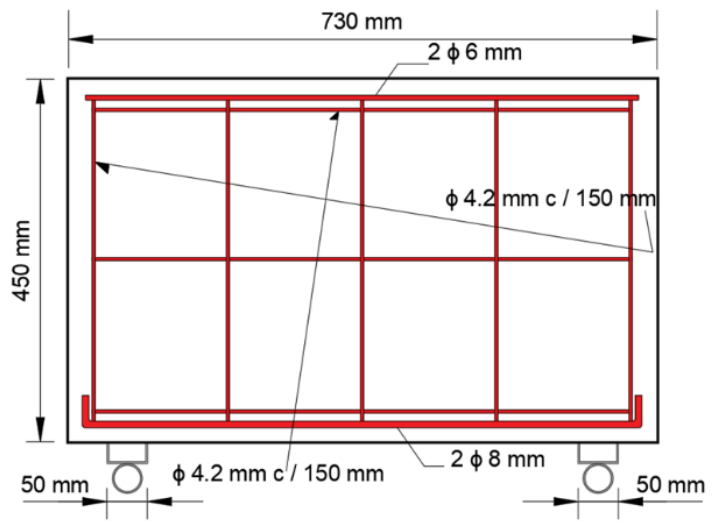

a)

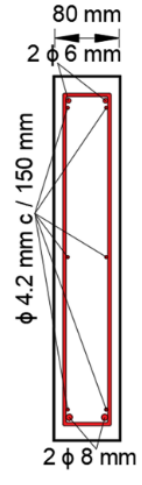

Figura 1 - Dimensiones geométricas y detalle de armaduras de los especímenes ensayados: a) Viga con armadura de corte; b) Viga sin armadura de corte

Tabla 1 - Detalle de los especímenes ensayados con su denominación y características

\begin{tabular}{|c|c|c|c|c|c|c|c|}
\hline Viga & $\begin{array}{c}\text { Ancho b } \\
(\mathbf{m m})\end{array}$ & $\begin{array}{c}\text { Altura } \mathbf{h} \\
(\mathbf{m m})\end{array}$ & $\begin{array}{c}\mathbf{d} \\
\mathbf{( m m})\end{array}$ & $\begin{array}{c}\boldsymbol{\rho} \\
\mathbf{( \% )}\end{array}$ & $\begin{array}{c}\boldsymbol{\rho}_{\boldsymbol{v}} \\
\mathbf{( \% )}\end{array}$ & $\begin{array}{c}\boldsymbol{\rho}_{\mathbf{h}} \\
\mathbf{( \% )}\end{array}$ & $\begin{array}{c}\mathbf{V}_{\mathbf{f}} \\
\mathbf{( \% )}\end{array}$ \\
\hline V1 & 80 & 450 & 426 & 0.29 & - & - & - \\
\hline V2 & 80 & 450 & 426 & 0.29 & 0.29 & 0.17 & - \\
\hline V331 & 80 & 450 & 426 & 0.29 & - & - & 0.31 \\
\hline V464 & 80 & 450 & 426 & 0.29 & - & - & 0.64 \\
\hline V531 & 80 & 450 & 426 & 0.29 & - & - & 0.31 \\
\hline V664 & 80 & 450 & 426 & 0.29 & - & - & 0.64 \\
\hline
\end{tabular}

\subsection{Características y propiedades de los materiales}

En la ejecución de las vigas se utilizaron dos tipos de hormigón, hormigón simple y HRFA, dosificados según el Método ICPA de Diseño Racional de Mezclas de Hormigón del Instituto del Cemento Portland Argentino (ICPA) en función de obtener una resistencia característica de rotura a compresión a los 28 días de $30 \mathrm{MPa}$. Se trabajó con agregados de la zona y cemento portland compuesto (CPC40). Para el HRFA se emplearon 
fibras de acero con extremo de gancho WIRAND ${ }^{\circledR}$, denominadas FF1, de $50 \mathrm{~mm}$ de longitud y $1 \mathrm{~mm}$ de diámetro. Las fibras se agregaron en forma manual, gradual y directamente a la mezcladora. En la Tabla 2 se presentan las propiedades mecánicas (resistencia media $f_{m}^{\prime}$ y módulo elástico E) de los hormigones, la tensión de fluencia y la resistencia a tracción $\left(f_{y}\right.$ y $\left.f_{t}\right)$ del acero de refuerzo y de las fibras.

Tabla 2 - Propiedades mecánicas de los materiales

\begin{tabular}{|c|c|c|c|c|c|}
\hline \multicolumn{2}{|c|}{ Materiales } & $\boldsymbol{f}_{\boldsymbol{m}}^{\prime}$ (MPa) & $\mathrm{E}(\mathrm{GPa})$ & $\boldsymbol{f}_{\boldsymbol{y}}(\mathrm{MPa})$ & $\boldsymbol{f t}(\mathrm{MPa})$ \\
\hline \multirow{2}{*}{ Hormigón } & Sin fibras & 33.25 & 27.10 & - & \\
\cline { 2 - 6 } & Con fibras & 35.60 & 28.04 & - & \\
\hline \multicolumn{2}{|c|}{ Acero } & - & 210 & 420 & 500 \\
\hline \multicolumn{2}{|c|}{ Fibras FF1 } & - & - & 800 & 1100 \\
\hline
\end{tabular}

\subsection{Procedimiento de ensayo e instrumentación}

Todos los ensayos realizados consistieron en la aplicación de cargas cuasi estáticas monótonas crecientes en el centro de la luz. Se adoptó una velocidad de carga de $1.5 \mathrm{~mm} / \mathrm{min}$. Se midieron cargas y desplazamientos en la dirección longitudinal y transversal. La carga fue aplicada usando una máquina de ensayos universal con sistema hidráulico con posibilidad de desarrollar una carga vertical nominal máxima de $1000 \mathrm{kN}$. Para la medición de los desplazamientos se utilizó un reloj comparador digital con una sensibilidad de $0.001 \mathrm{~mm}$. En la Figura 2 se muestra el esquema de ensayo e instrumentación utilizado. El instrumento de medición de los desplazamientos transversales fue retirado antes de alcanzar la carga máxima, como medida de precaución para evitar la posible rotura de este.

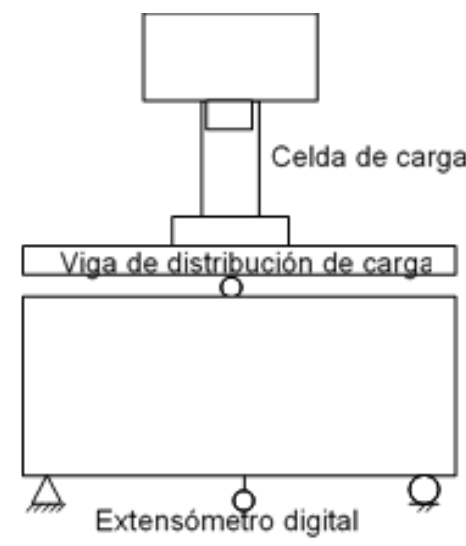

Figura 2 - Esquema de ensayo e instrumentación

\section{Resultados experimentales}

\subsection{Cargas últimas y curvas carga- desplazamiento}

En Tabla 3 se presentan los resultados obtenidos durante los ensayos, correspondientes a las seis vigas consideradas en este estudio. Se muestran cargas últimas, incremento de resistencia en comparación con el espécimen V1 (hormigón simple sin armadura de corte) y modos de falla.

Según se puede ver en la Tabla, la viga con armadura de corte y los especímenes de HRFA muestran un incremento significativo en resistencia, en comparación con la viga V1, de hormigón simple, con armadura de flexión solamente. Sin dudas, la viga V2 con armadura de corte mínima, registra el mayor incremento de carga, y como se observa en la Figura 3, la mayor ductilidad. 
Table 3 - Resultados experimentales

\begin{tabular}{|c|c|c|c|}
\hline Viga & Pu (kN) & Incremento de resistencia (\%) & Modos de falla \\
\hline V1 & 124.40 & - & Falla en apoyo \\
\hline V2 & 222.20 & 78.6 & Corte \\
\hline V331 & 185.00 & 48.7 & Corte \\
\hline V464 & 208.50 & 67.6 & Corte \\
\hline V531 & 171.92 & 38.2 & Aplastamiento del hormigón \\
\hline V664 & 210.48 & 69.2 & Corte \\
\hline
\end{tabular}

En la Figura 3 se reproducen las curvas carga-desplazamiento axial y transversal (P- $\delta l ; P-\delta t)$ de las vigas de hormigón simple, con y sin armadura de corte, y de las vigas de HRFA. Los especímenes de HRFA y $0.64 \%$ de fracción de volumen de fibras, alcanzaron un valor de carga última similar (210.48 kN y 208.50 kN) y muy cercano a V2 (222.20 kN). En las VGA de HRFA con una fracción de volumen de fibras de $0.31 \%$, los valores de carga última fueron menores comparados con los obtenidos por la viga V2 y los especímenes de HRFA y $0.64 \%$ de $V f$. Sin embargo, dichos valores resultaron un $48.7 \%$ y un $38 \%$ mayores que la resistencia máxima de la viga V1. El incremento de la fracción de volumen de fibras se reflejó en un aumento de la resistencia al corte. Sin embargo, se debe tener en cuenta que un volumen alto de fibras (mayor a $1.5 \%$, según Adam et al. (2016)) puede afectar la trabajabilidad de la mezcla.

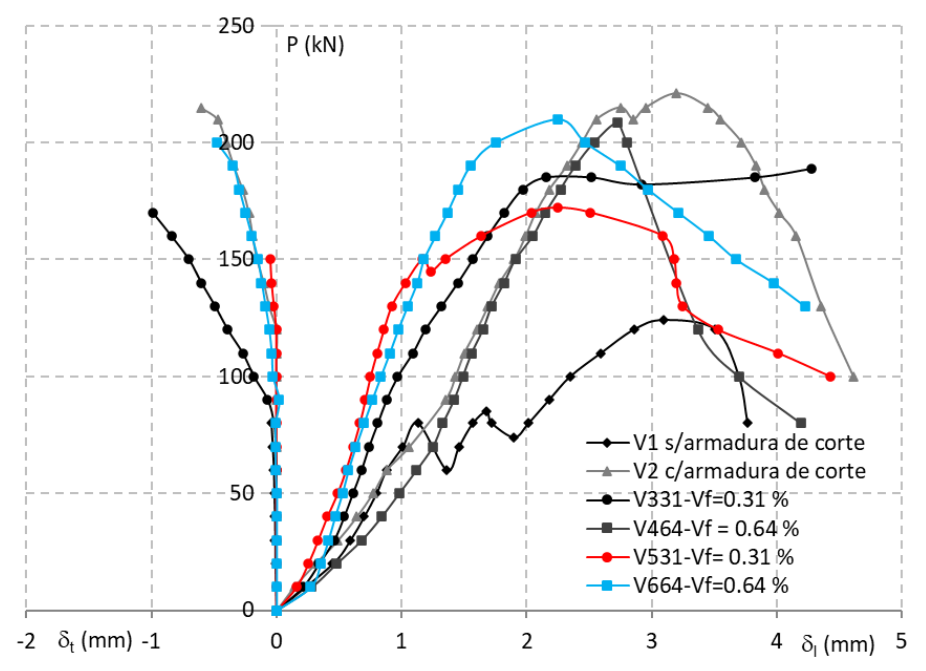

Figura 3 - Curvas carga-desplazamiento axial y transversal (P- $\delta l$ y $P-\delta t)$

La capacidad de deformación de las VGA de HRFA fue menor que la registrada por la viga con armadura de estribos (V2), mientras que la rigidez, en la mayoría de los casos resultó mayor. La incorporación de fibras de acero mejoró el comportamiento en resistencia de las vigas sin armadura de estribos verticales $y$ horizontales, pero no logró reemplazar dichos estribos.

\subsection{Modos de falla}

En las Figuras 4 a) se muestra el modo de falla de la viga V2 con armadura de corte mínima. Con el incremento de la carga, la fisura diagonal se extiende hacia los puntos apoyo y de carga, se aumenta el espesor de las fisuras existentes y en la etapa final que conduce a la falla por corte, se produce la rotura del hormigón situado por encima de la fisura crítica de corte. En el caso de la viga V1, de hormigón simple y sin armadura de corte, se produjo la rotura en uno de los apoyos debido al anclaje deficiente de la armadura longitudinal. En la Figura 4 b) se observa la falla por corte, si rotura del hormigón, del espécimen V664. 


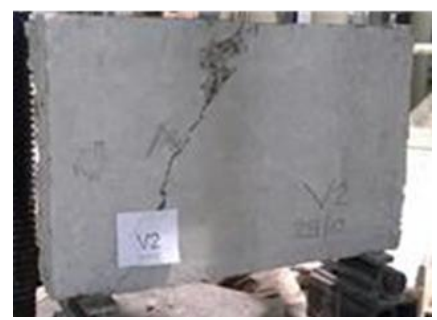

a)

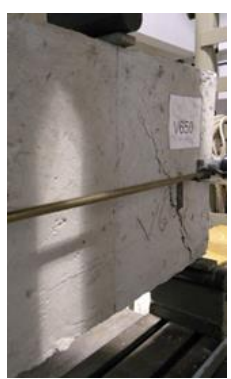

b)

Figura 4 - Modos de falla de las VGA: a) Viga V2 sin fibras, con estribos; b) Viga de HRFA, V664

\section{Análisis Teórico}

En la Tabla 4 se comparan los valores experimentales de carga última Pu de las VGA de hormigón simple y hormigón armado, con los obtenidos a partir del método puntal tensor que recomienda para el cálculo el CIRSOC 201-05 (2005) y también con modelos teóricos propuestos por otros autores, Arabzadeh et al. (2009) y Matamoros y Wang (2003) que surgen de modificaciones realizadas al modelo puntal tensor original. Para las VGA de HRFA se utilizan los mismos modelos. Sin embargo, ninguno de los modelos presentados contempla la presencia de las fibras de acero en sus formulaciones.

Table 4 - Comparación de resultados experimentales y teóricos

\begin{tabular}{|c|c|c|c|c|}
\hline Viga & Pu exp. (kN) & $\begin{array}{c}\text { Pu } \text { MPT (kN) } \\
\text { (CIRSOC 201-05) }\end{array}$ & $\begin{array}{c}\text { Pu (kN) } \\
\text { (Arabzadeh et al.) }\end{array}$ & $\begin{array}{c}\text { Pu (kN) } \\
\text { (Matamoros y Wong) }\end{array}$ \\
\hline V1 & 124.40 & 88 & 108,30 & 160 \\
\hline V2 & 222.20 & 88 & 108.50 & 205 \\
\hline V331-V531 & $185.00-171.92$ & 88 & 113 & 216 \\
\hline V464-V664 & $208.50-210.48$ & 88 & 113 & 216 \\
\hline
\end{tabular}

Se puede ver en la Tabla, que tanto el MPT, como el de Arabzadeh et al. (2009) dan resultados conservadores. El modelo de Matamoros y Wong tiene una mejor correlación con el valor experimental de la VGA V2 (con estribos) pero sobrestima el de la VGA V1 sin armadura de corte y los resultados correspondientes a los especímenes de HRFA.

\section{Simulación numérica}

Con el objetivo de predecir numéricamente la capacidad resistente de las VGA de hormigón y HRFA correspondientes a la campaña experimental descripta en apartados anteriores, se simuló el ensayo a flexión en 3 (tres) puntos mediante un modelo tridimensional. Dicho modelo fue implementado en un software de análisis por elementos finitos ABAQUSR. Para representar el comportamiento del hormigón simple se adoptó el modelo "Concrete Damaged Plasticity"(CDP), Abaqus Analysis User's Guide (2017). El CDP supone que los principales mecanismos de falla del hormigón son fisuración por tracción y aplastamiento por compresión. Así, el modelo considera diferentes comportamientos bajo estas dos solicitaciones.

Para el HRFA, se adoptaron curvas tensión-deformación que permitieron definir su comportamiento a compresión y a tracción. Con respecto al comportamiento a compresión se utilizó un modelo desarrollado por Barros y Figueras (1999). Para el aporte de las fibras a la resistencia tracción se tuvieron en cuenta el modelo de Voo y Foster (2003) y Gouveia et al. (2014). En base a esas formulaciones y los datos obtenidos 
experimentalmente, se obtuvieron curvas tensión deformación a tracción utilizadas en el modelo CDP para HRFA.

Para los refuerzos de acero, se adoptó el modelo de material de tensión-deformacion elásticoperfectamente plástico.

\subsection{Modelo de elementos finitos del ensayo a flexión VGA.}

El elemento estructural se consideró simplemente apoyado y con una carga puntual en la cara superior, en el centro de la luz entre los apoyos. Para modelar el hormigón se utilizaron elementos sólidos 3D de 8 nodos de integración reducida denominado C3D8R. Para el caso de las barras de acero de refuerzo se utilizaron elementos lineales de dos nodos "2-node linear 3-D truss" (T3D2). En la Tabla 5 se muestra un esquema de la malla de elementos finitos utilizada y las condiciones de contorno.

En la Tabla 5 se muestran los valores de carga última obtenidos para los distintos especímenes simulados, juntamente con los valores experimentales promedio y el error relativo a los efectos de la validación del modelo. Se observa que los resultados numéricos aproximan razonablemente los valores de $P_{u}$ obtenidos experimentalmente.

Tabla 5 - Valores de carga última experimentales y numéricos.

\begin{tabular}{|c|c|c|c|c|}
\hline Viga & Pu exp. (kN) & Pu Numérico (kN) & Error relativo (\%) & Malla de EF \\
\hline V1 & 124 & 151.1 & 21.85 & \multirow{2}{*}{} \\
\hline V2 & 222 & 214.4 & 3.50 & \multirow{2}{*}{} \\
\hline V331-1 Y V531-2 & 178.46 & 198 & 11.00 & \\
V464-1 Y V664-2 & 209.49 & 212.7 & 1.53 & \\
\hline
\end{tabular}

\section{Conclusiones}

Si bien es necesario un mayor número de ensayos, de acuerdo con los resultados experimentales, teóricos y numéricos obtenidos en este estudio se pueden enunciar las siguientes conclusiones:

Las VGA de HRFA tuvieron un comportamiento muy satisfactorio en cuanto a resistencia y ductilidad, en comparación con la viga de hormigón simple sin armadura de corte.

Con el aumento del volumen de fibras, se incrementó la capacidad resistente de las VGA, obteniéndose valores cercanos al alcanzado por el espécimen con armadura de estribos y sin fibras. Sin embargo, y dado que el número de ensayos realizados hasta el momento es pequeño, no se puede pensar aún en un reemplazo parcial y/o total de la armadura tradicional de corte, por HRFA.

Todas las vigas de HRFA tuvieron un tipo de falla dúctil, conservando el monolitismo luego de la falla.

La comparación de los valores de carga última obtenidos experimentalmente con las predicciones resultantes de la aplicación de modelos teóricos, MPT y modificaciones a dicho método, muestra que dichos modelos, en general, dan resultados conservadores y no contemplan el aporte de las fibras de acero.

El análisis de elementos finitos no lineal pudo predecir razonablemente el valor de carga última de todas las VGA de HRFA, con diferencias de entre el $1.53 \%$ y el $22 \%$.

Se debe destacar que actualmente se está estudiando un modelo no homogéneo que considera por separado el hormigón y las fibras. Además, y para validar dicho modelo, se está llevando a cabo una campaña experimental donde se estudian otros volúmenes de fibras de acero y la combinación con fibras plásticas. 


\section{Agradecimientos}

Los autores del trabajo agradecen a la Universidad Tecnológica Nacional, FRCU, por el apoyo económico brindado para la realización de la campaña experimental y al jefe de laboratorio de Ingeniería Civil de la FRCU, Ingeniero Ricardo Gómez, por su valiosa asistencia y colaboración en la realización de los ensayos.

\section{References}

Abaqus Analysis User's Guide. Volume 3: Materials. (2017). Dessault Systems Simulia Corporation.

ACl 318S-14 (2014). Requisitos de Reglamento para Concreto Estructural.

ACI 544.1R (1996-2002). Report on Fiber Reinforced Concrete.

Adrija, D., Geevar, I., Menon, D., Prasad, A. (2017) Assessment of strut-and-tie methods to estimate ultimate strength of RC deep beams. Proceedings of Int. Multiconference of Eng. and Comp. Scientists, IMECS 2017, Vol II.

Ahmad, S., Shah, A., Zaman, M., Salimullah, K. (2011). Design and evaluation of the shear strength of deep beams by strut and tie model (STM). IJST, Transactions of Civil and Environmental Engineering, 35, 1-13.

Arabzadeh, A., Rahaie, A., Aghayari, R. (2009). A Simple Strut-and-Tie Model for Prediction of Ultimate Shear Strength of RC Deep Beams. International Journal of Civil Engineering,7(3), 141-153.

Beshara, F., Mahmoud, A., El-Barbary, A. (2015). Experimental Behavior of Steel Fiber Reinforced Concrete Deep Beams. ERJ- Faculty of Engineering at Shoubra, 104-115.

Chen, H., Yi, W., Hwang, H. (2018). Cracking strut-and-tie model for shear strength evaluation of reinforced concrete deep beams. Engineering Structures, 163, 396-408.

Chetchotisak, P., Teerawong, J., Chetchotsak, D., Yindeesuk, S. (2014). Efficiency Factors for Reinforced Concrete Deep Beams: Part 1- Improved models. Advanced Materials Research, 931-932, 506-513.

CIRSOC 201-2005. (2005) Reglamento Argentino de Estructuras de Hormigón.

Gouveia N., Fernandes N., Faria D., Ramos A., Lúcio V. (2014). SFRC flat slabs punching behaviour experimental research. Composites: Part B, 63, 161-171.

Matamoros, A. y Wong, K. (2003). Design of Simply Supported Deep Beams Using Strut-and-Tie Models. ACI Structural Journal, 100 (6), 704-712.

Moradi, M. y Reza Esfahani, M. (2017). Application of the strut-and-tie method for steel fiber reinforced concrete deep beams. Construction and Building Materials, 131, 423-437.

Naik, U. y Kute, S. (2017). Use of Steel Fibers as Shear Reinforcement for Deep Beams in Shear: an experimental study. International Journal of Engineering Science and Computing, 7, 13748-13750.

Orler, R. y Donini, H. (2008). Diseño básico de hormigón estructural. Editorial Científica Universitaria.

Pabale A. y Mehetre A.J. (2015). Evaluation of Effectiveness of SFRC Deep Beams in Shear. International Journal of Research Studies in Science, Engineering and Technology, 2, 120-124.

Ruano, G. (2013). Refuerzo de elementos estructurales de hormigón con hormigón reforzado con fibras. Tesis Doctoral. Instituto de Estructuras “Ing. Guzman”, Universidad Nacional de Tucumán, Argentina.

Shah, A. y Ribakov, Y. (2011). Recent trends in steel fibered high-strength concrete. Materials and Design, 32, 4122-4151.

Voo, J. y Fooster, S. (2003). Variable engagement model for fibre-reinforced concrete in tension. School of Civil \& Environmental Engineering.

Zhang, N. y Tan, K. (2007). Direct strut-and-tie model for single span and continuous deep beams. Engineering Structures, 29, 2987-3001. 